\section{Sicherheit in der Cloud}

\author{
Bei der Auslagerung von IT-Diensten wird die Cloud immer \\ wichtiger. Doch Unternehmen haben Sicherheitsbedenken.
}

Das Thema Cloud Computing polarisiert: In seinem Cloud-Monitor 2013 kommt der Branchenverband Bitkom zu dem Schluss, dass der Markt in einen gewissen Reifeprozess eingetreten ist. Während eine wachsende Zahl von Auftraggebern positive Erfahrungen mit dem Auslagern von Diensten in die Private und/oder Public Cloud gemacht hat und diese Alternative zum Inhouse-Betrieb weiter nutzen möchte, nehmen auch die Cloud-Skeptiker zu - geschrumpft ist hingegen der Anteil der Unentschlossenen. Dabei fällt auf, dass große Unternehmen tendenziell offener an das Thema herangehen als kleine und mittelständische Unternehmen. Das dürfte nicht zuletzt daran liegen, dass der Markt für Cloud-Services in raschem Wachstum begriffen und die Vertrauenswürdigkeit der einzelnen Anbieter für Auftraggeber nur schwer zu überprüfen ist, sofern man kein ausgesprochener Spezialist ist. Die Orientierung erleichtern könnte eine unabhängige und neutrale Zertifizierung von Cloud-Services.

Mehr dazu finden Sie auf:

[Sfip] $^{*}$ www.springerprofessional.de/4943510

\section{Vom Netzwerk profitieren}

\section{Mithilfe von Netzwerkorganisationen können Unternehmen projektweise auf externes Spezialwissen zugreifen.}

Über viele Jahre verzeichneten die Management-Literatur und der betriebliche Alltag nur zwei wesentliche Organisationsformen: die funktionale und die divisionale Organisation. Mit dem Trend zur Wissensgesellschaft, dem Siegeszug des Internets und der Konzentration vieler Unternehmen auf den Kern ihres Geschäftes setzt sich nun auch die Netzwerkorganisation in etlichen Branchen durch. Nach Meinung von Experten gehört dieser Organisationsform sogar die Zukunft, weil sie hierarchische Elemente der Koordination mit Marktelementen intelligent verknüpft. Unter Netzwerkorganisationen verstehen Wissenschaftler den Zusammenschluss von autonomen, meist auch unternehmerisch selbstständigen Experten für die Durchführung eines gemeinsamen Projektes. Unternehmen können so auf externes Expertenwissen zurückgreifen, ohne dass sie diese Kompetenzen aufwendig und kostenintensiv selber aufbauen müssten. Die Experten arbeiten meist an verschiedenen Arbeitsplätzen, in verschiedenen Ländern und schließen sich im Internet zu einem Wissensnetzwerk zusammen. Mehr dazu finden sie auf:

SfP $^{*}$ www.springerprofessional.de/4921022
Wesentliche Erfolgsfaktoren der Führung

$\pi$ springer-gabler.de

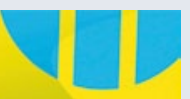

Auf der Suche nach Führungsidentität

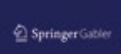

Günter Bolten

Auf der Suche nach Führungsidentität Orientierungshilfen für Führungskräfte

2013. VII, 185 S. 19 Abb. Br.

$€$ (D) 39,99

ISBN 978-3-658-01108-6

Zahlreiche Bücher befassen sich mit dem Thema Führung, doch keines beleuchtet den Aspekt der Identifikation der Mitarbeiter mit ihren Vorgesetzen so intensiv wie das von Günter Bolten. Dabei werden Führungskräfte umso überzeugender und erfolgreicher, je besser es innen gelingt, eine Identifikationswirkung bei ihren Mitarbeitern zu erzeugen. Je stärker sich Menschen mit ihren Aufgaben und Vorgesetzten identifizieren, desto größer werden Engagement und Erfolgsaussichten. Führungsidentifikation wird somit zu einem zentralen Element erfolgreicher Mitarbeiterführung.

\section{Einfach bestellen:}

SpringerDE-service@springer.com Telefon +49 (0)6221/345- 4301 